\title{
Inflation in a Renormalizable Cosmological Model and The Cosmic No Hair Conjecture
}

\author{
Kei-ichi Maeda \\ Dept. of Physics, Unizersity of Tokyo, \\ Bunkyo-ku, Tokyo 119, JAPAN \\ Jaime A. Stein-Schabes \\ NASA/Fermilab Astruphysics Center \\ MS 209 Fermi National Accelerator Laboratory \\ P. O. Box 500 Batavio, Mlinois 60510 \\ and \\ Toshifumi Futamase \\ Dept. of Physics, Hirosaki University \\ Hirosaki, JAPAN

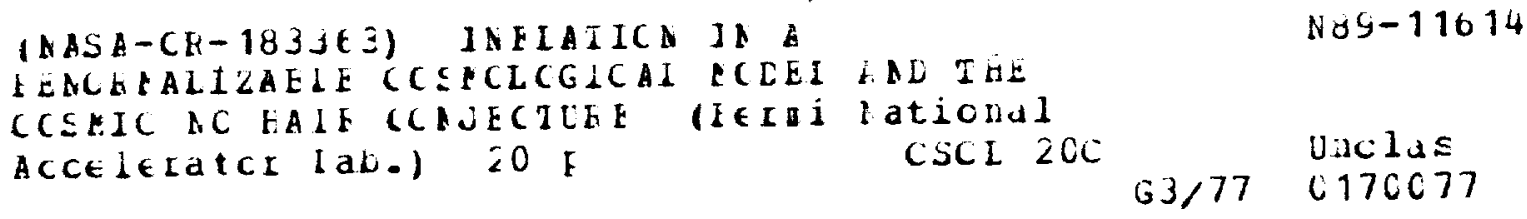

\begin{abstract}
:
The possibility of having inflation in a renormalizable cosmological model is investigated. The Cosmic No Hair Conjecture is proved to hold for all Bianchi types except Bianchi $I X$. By the use of a conforma transformation on the metric we show that these models are equivalent to the ones described by the Einstein-Hilbert action for gravity miuimally coupled to a set of scalar fields with inflationary potentials. Henceforth, we prove that inflationary solutions behave as attractors in solution space, making it a natural event in the evolution of such models.
\end{abstract}




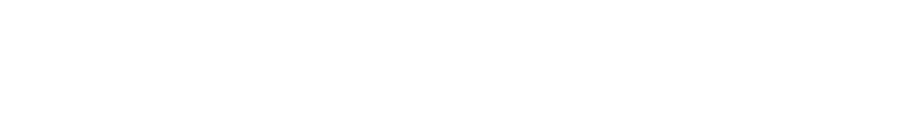

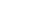




\section{Introduction}

Inflation has become one of the most desira ole features of any cosmological model. There are several reasons for this, ranging frorı particle physics considerations to the large scale structure of the spacetime around $u$. Since most of these issues have been extensively discussed in the literature we shall just refer the reader to the relevant source [i].

One amongst many problems solved by inflation is that of explaining the isotropy and large scale homogeneity of the observable part of the Universe $\left(H_{0}^{-1} \sim 10^{28} \mathrm{~cm}\right)$. As a bonus inflation provides us with a mechanism to oroduce density perturbations that could be responsible for the formation of structure in $t$ se Universe after inflation (see Turner in [1]).

At the moment there are several models fir inflation, the so called new inflation proposed by Albrecht and Steinhardt [1], the chrotic inflation proposed by Linde [1] and the higher derivative gravity models [2], [3]. The first two models are based on a rather similar theory, namely that of gravity coupled minimally to a real scalar field. Even though the ideas behind these two theories are rather different, in practice one of the few differences is the form of the potential on which the scalar field moves (e.g. ColemanWeinberg type vs. $m^{2} \phi^{2}$ or $\left.\lambda \phi^{4}\right)$. The important feature of these two models is the existence of a very small coupling constant (s:e Turner in [1]). However, the chaotic scenario seems to be more natural in the sense that the form of the potential needed is more generic and the restrictions imposed on the initial conditions for the fields are less severe. The third model is substantially differert, both in principle and in practice. It is based on adding extra terms, proportional to tie Piemann tensor square and some of its contractions, to the standard Einstein-Hilbert action. The appeal of this theory resides in the fact that it contains inflationary solutior s without having an inflaton scalar field $[2]$ - [4]. Inflation is then a consequence of (almsst) pure gravitational interactions. 


\section{ORIGINAL PAGE IS \\ OF POOR QUALITY}

In the first two theories inflation appears as a generic feature, not only when the spacetime is the familiar flat or open Robertson-Walker but even when anisotropic case are considered [8]. These results have been extended to a large class of inhomogeneous open or flat models (nonpositive three curvature i.e. ${ }^{3} R \equiv P \leq 0$ ) for the case of new inflation [9], and for the $R^{2}$ model [4-7]. We would like to add that some arguments have been given in support of the idea that closed models will also inflate unless their spatial topology is $S^{3}$ or $S^{2} \times S^{1}$ (see Barrow in [7]). This would certainly enlarge, and contribute to the measure of the set of models that undergo inflation [10].

The motivation for any of the above theories is understandable, almost all theories of the Universe contain at least one scalar field and gravity. The usual argument for adding higher derivative terms to the gravitational action is the renormalizability of the theory [11]. The divergences in the gravitational action to first order are proportional to $R^{2}, R_{\mu \nu} R^{\mu \nu}$ and $R_{\mu \nu \rho \sigma} R^{\mu \nu \rho \sigma}$ so it is only natural to add these terms in order to renormalize the theory. On the other hand we know of the existence of scalar fields in the theory so it seems only natural to have at least one of these. The renormalizability of the theory demands an additional interaction term between the graviton and the scalar field of the form $-\frac{1}{2} \xi \phi^{2} R[11]$. The lagrangian can then be written as (see Brown and Collins in [11])

$$
\mathcal{L}=\mathcal{L}_{g}+\mathcal{L}_{\phi}+\mathcal{L}_{g \phi}
$$

where $\mathcal{L}_{\rho}$ and $\mathcal{L}_{\phi}$ are the gravitational and scalar field lagrangians respectively and $\mathcal{L}_{g \phi}$ represents the interaction term. These are given by

$$
\begin{aligned}
\mathcal{L}_{g}= & \Lambda+\kappa_{0} R+a_{0}\left(R^{2}-4 R_{\mu} R^{\mu \nu}+R_{\mu \nu \mu \sigma} R^{\mu \nu \rho \sigma}\right) \\
& \quad+b_{0} C_{\mu \nu \rho \sigma} C^{\mu \nu \rho \sigma}+c_{0} R^{2} \\
\mathcal{L}_{\phi}= & -\frac{1}{2}(\nabla \phi)^{2}-V(\phi) \\
\mathcal{L}_{g \phi}= & -\frac{1}{2} \xi \phi^{2} R
\end{aligned}
$$


where $V(\phi)$ is some arbitrary renormalizable potential, and $\Lambda, \kappa_{0}, \xi, a_{0}, b_{0}, c_{0}$ are constants (satisfying the renormalization group equations 11 )). $\kappa_{0}^{-1}$ is proportionai to $16 \pi G_{N}$, but for practical purposes we will let the proportior ality factor unspecify. $C_{\mu \nu \rho \sigma}$ is the Weyl tensor and will vanish for conformally flat metric: (like in the RW case). The quantity multiplying $a_{0}$ is the Gauss-Bonnet density, when iategrated over the invariant four-volume $\sqrt{-g} d^{4} x$ it gives a topological invariant, so its variation vanishes and as a consequence it does not contribute to the equations of motion for this reason we snall set $a_{0}=0$ (see Barth and Christensen in [11]).

The theory described by the above equations is very general and it contains many special cases that have been studied in the past. Since all of these are important in their own right we have compiled two tables of referen es containing most of these and indicated whether these undergo inflation. The cases have been separated into two classes. Table one represents purely gravitational models, i.e. $\mathcal{L}_{\delta \phi}=\mathcal{L}_{\phi}=0$, while table two comprises the cases where a scalar field is present. The sta 1 dard case $a_{0}=b_{0}=c_{0}=\xi=0$ has been omitted from the table while those marked witl ${ }^{* * *}$ are the ones studied in this paper.

We would like to comment that setting $\kappa_{0}=0$ does not mean we are setting the gravitational constant to zero, but rather that , ither at some stage in the early universe the quadratic terms or the $\xi \phi^{2} R$ terms are the dominant ones in the action, or that gravity is induced by a symmetry breaking meclanism where $\phi$ acquires a non zero VEV determining the effective gravitational constant [12].

The paper will be organize as follows. In Scc. 2. we will review the induced gravity case and by the use of the ccuformal transformation recast its resuits and consequences, we could think of this as the case $\left(\kappa_{0}=0\right)$. In Sec 3 . we shall study a much more general cas:. We shall finalize with some comments and conclusiurs 


\section{ORIGINAL PAGE IS}

OF POOR QUALITY

\section{Induced Gravity Model}

This model was first proposed by Zee [12] as an attempt to use symmetry breaking to generate the Einstein term in the action at low energies. The model is described by a lagrangian of the form

$$
\mathcal{L}=\frac{1}{2} \epsilon \phi^{2} R-\frac{1}{2}(\nabla \phi)^{2}-V(\phi)
$$

where $V(\phi)=\frac{\lambda}{8}\left(\phi^{2}-\eta^{2}\right)^{2}$ and $8 \pi \epsilon \equiv-\xi$ is positive. In this model the present gravitational constant would be given by the VEV of the scalar field, i.e. $\left.G_{N}=(\epsilon<\phi\rangle^{2}\right)^{-1}$. The existence of inflationary solutions had already been noticed by Acceta et al [12]. They found that exponential and power law inflation occured for $\phi \rightarrow \infty$ and $\phi \rightarrow 0$ respectively for not very special values of $\epsilon$ and $\lambda$.

We will now show, using the conformal transformation technique, why is it that this model inflates naturally in the isotropic case and subsequently generalize this result to the anisotropic models. By conformally transforming the metric, the action can be rewritten as the Einstein-Hilbert action in minimal coupling with a scalar field (this was first done for the $R+\epsilon R^{2}$ model by Whitt [17]). The advantage of this transformation is clear, we can andyze the behaviour of the scalar field simply by looking at the potential in which it moves.

Let us consider the following conformal transformation:

$$
\hat{g}_{\mu \nu} \equiv \kappa^{2} \epsilon \phi^{2} g_{\mu \nu}
$$

In terms of this neiv metric tho action coming from (2.1) becomes

$$
\begin{gathered}
S=\int d^{4} x \sqrt{-\hat{g}}\left[\frac{1}{2 \kappa^{2}} R(\hat{g})-\frac{1}{2}(\hat{\nabla} \psi)^{2}-\hat{V}(\psi)\right] \\
\psi \equiv \frac{\sqrt{6+\frac{1}{e}}}{\kappa} \ln \phi
\end{gathered}
$$




$$
\begin{gathered}
\text { ORIGN - POE :S } \\
\text { OF POO \& QUALIT } \\
\hat{V}(\psi) \equiv \frac{\lambda \eta^{4}}{8 \kappa^{4} \epsilon^{2}}\left[\frac{1}{\eta^{2}}-\operatorname{xp}\left(-2 \sqrt{\frac{\epsilon}{1+6 \epsilon}} \kappa \psi\right)\right]^{2}
\end{gathered}
$$

Fig.1 shows the potential $\hat{V}(\psi)$ for $\eta=1, \epsilon=\frac{1}{4}, \kappa=1$. It is easy to understand why the model undergoes exponential inflation for lirge positive $\psi(\phi \rightarrow \infty)$, the potential is so flat the that model behaves as if dominated by an effective cosmological constant $\Lambda_{e f f}=\frac{\lambda}{8 \kappa^{+} e^{2}}$, producing the usual slow rolling of the $\psi$ field. In these regime we can transform back to the real spacetime where $\phi \mathrm{l}$ ives and see that inflation takes place as well. Following the exact same logic we deduce that power law inflation is also possible when $\psi$ is a large negative number $(\phi \rightarrow 0)$. The asymptotic form of $\hat{V}(\psi)$ is given by

$$
\hat{V} \sim \exp \left(-4 \sqrt{\frac{\epsilon}{1+6 \epsilon}} \kappa \psi\right) \quad \text { for } \quad \psi \rightarrow-\infty
$$

then provided $\epsilon<\frac{1}{2}$ ( the exponent is smaller than $\sqrt{2}$ ) power law inflation will occur. The condition on $\epsilon$ ensures that the potential is not too steep. Power-law inflation in the fictitious $\hat{g}$-world will always guarantee power-la $w$ inflation in the real $g$-world, we can see this by setting the scale factor $\hat{a} \propto \hat{t}^{\hat{p}}$ then $a \propto{ }^{p}$ and if $\hat{p}>1$ then $p=2 \hat{p}-1>1$.

Let us now turn to the anisotropic cases. We could of course try to prove the generality of the result by direct calculation, but this coulc be extremely difficult and time consuming. Instead, we shall use a shortcut. In order t') go from one representation to the other we have not specified the form of the metric $g_{\mu \nu}$ (or $\hat{g}_{\mu \nu}$ ) so we can obtain our results in either world and transform it back. Notice that in the $\hat{g}$ world the energy-momentum tensor for the scalar field subtracting $\Lambda_{e f f}$ satisfies the dominant and weak energy conditions. Hence, we can apply Wald's cosmic no hair conjecture [8] and conclude that inflation will always take place (except maybe in Biancli $1 X$ ). This result can be further extended to a class of :nhomogeneous spacetimes with nu npositive three curvature. Inflation is a natural event if the universe starts out from a r"gion of large positive $\phi$. If, on the other hand $\phi \rightarrow 0(\psi \rightarrow-\infty)$, we can use a similar result found by Moss and Sahni [8] for the case of power law inflation (unfortunately this result only applies to the case of a massive 
scalar field) to infer the generality of the event.

\section{Renormalizable Model}

In this section we shall consider a more general case. The model is described by eq. (1.1) with $b_{0}=0$,

$$
\mathcal{L}=\frac{1}{2 \kappa^{2}} R-\frac{1}{2} \xi \phi^{2} R+\frac{\alpha}{2 \kappa^{2}} R^{2}-\frac{1}{2}(\nabla \phi)^{2}-V(\phi)
$$

where a cosmological constant $\Lambda$ has been included in $V(\phi)$.

As in $\S .2$, we will use a conformal transformation to reduce the system (1.1) to that of gravity coupled to two scalar fields. Such a system is easy to analyze. We need only to know the form of the potential to deduce the dynamical behaviour of the scalar fields.

We will outline the method for finding the conformal transformation that will do the trick. Firstly, write down the basic equations coming from the variation of (1.1), then perform an arbitrary conformal transformation of the form

$$
\hat{g}_{\mu \nu}=e^{2 \omega\left(x^{\rho}\right)} g_{\mu \nu}
$$

where $\omega$ is an unknown function to be determined later. Secondly, calculate the second order derivative of $R$ and $\omega$, identify these terms so as to eliminate them from the equations and finally write the leftover as "Einstein's" equations with some scalar fields. This technique can be used for an even more general case [14].

Following the above method, we find

$$
\omega=\frac{\kappa}{\sqrt{6}} \psi \equiv \frac{1}{2} \ln \left[1-\kappa^{2} \xi \phi^{2}+2 \alpha R\right]
$$

and the equivalent action

$$
S=\int d^{4} x \sqrt{-\hat{g}}\left[\frac{1}{2 \kappa^{2}} R(\hat{g})-\frac{1}{2}(\hat{\nabla} \psi)^{2}-\frac{1}{2} e^{-\sqrt{\frac{2}{3}} \kappa \psi}(\hat{\nabla} \phi)^{2}-\hat{U}(\phi, \psi)\right]
$$


where the potential in the $\dot{g}$-world is

$$
\hat{U}(\phi, \psi) \equiv \frac{1}{8 \alpha \kappa^{2}}\left[1-\left(1-\kappa^{2} \xi \phi^{2}\right) 2^{-\sqrt{\frac{2}{3}} \kappa \psi}\right]^{2}+e^{-\sqrt{\frac{2}{3}} \kappa \psi} V(\phi)
$$

Now we shall analyse the potential $\hat{U}(\phi, \psi)$ in two different cases:

Case $\mathbf{A}: V(\phi)=0$

Let us focus our attention in the case $\xi>0$. The other case is rather similar so we will omit it. It is useful to define a critical value for the scalar field as

$$
\phi_{c} \equiv\left(\kappa^{2} \xi^{-1}\right.
$$

then the potential $\hat{U}(\phi, \psi)$ has a zero at

$$
\phi \equiv \phi_{0}=\phi_{c} \sqrt{1-\exp \left(\sqrt{\frac{2}{3}} \kappa \psi\right)}
$$

The potential is shown in Figs. 2a and 2b. Frcm this and Eq. (3.4) we find that there is a very flat plateau in the potential for large $\psi$, unless $\phi$ is much larger than $\phi_{c}$. The evolution of such a model might proceed as follo ws. Near the Planck scale the Universe is probably in an excited state and its energy is la ger than the plateau. We could imagine then the universe-particle hovering over the potential. As time goes by it might land on a region of large $\psi$. If so then it will effectivel $r$ becomes dominated by a cosmological constant, hence invoking the No Hair Conjecture, we would conclude that, if it is an open or flat Bianchi model (or a sufficiently constraint Bianchi $I X$ ), it will inflate. If $\xi>0$ but not to large, then the model will not inflat: (it might even land at the minimum of the potential, however we belive this is highly unlikely). It then continues on a slow roll towards the minimum inflating and becoming more isotropic and De Sitter-like as it goes along, ending completely isotropic at the bottom of the potential. The isotropization time is of order ore Hubble expansion tine $\hat{\tau} \equiv 2 \sqrt{6} \bar{x}$, when translated into the $g$-world, the time scale becomes

$$
\tau \equiv \exp \left(-\frac{\kappa}{\sqrt{ }}=\psi_{1}\right) \hat{\tau}
$$




\section{ORIGINAL PAGE IS \\ OF POOR QUALITY}

where $\psi_{1}$ is the value of $\psi$ when the universe becomes isotropic (see also [8]). Because of slow rolling during this period, the value of $\psi$ changes very slowly hence the definition of $\psi_{1}$ is not too ambiguous.

One interesting feature in this model must be mentioned: After the inflationary period has come to an end, the universe evolves towards the zero of the potential (as defined by (3.6). Moreover, from this eq. we find that the value of the $\phi$ field at zero is bounded by the critical value, i.e. $\phi_{0}<\phi_{c}$. This is interesting because in the anisotropic case a universe where gravity is not minimally coupled to a scalar field is bound to encounter a singularity at $\phi>\phi_{c}$. This effect was first discussed by Starobinskii [16]. However, if curvature square terms are taken into account (as in our model), then the universe always evolves into the region with $\phi<\phi_{c}$. Hence, even if the universe starts with $\phi>\phi_{c}$, it does reach its present state.

Case B : $V(\phi) \neq 0$

This case is a little more involved as it depends on the explicit form of $V(\phi) . \hat{U}(\phi, \psi)$ is depicted in Figs. 3a and $3 b$ for $V(\phi)=-\frac{1}{2} m^{2} \phi^{2}+\frac{1}{4} \lambda \phi^{4}$. Nevertheless, if the scale of the potential $V(\phi)$ is much smaller than $\dot{U}(\phi, \psi)$ (for example: $V(\phi)$ might be a GUT scale or below while $\hat{U}(\phi, \psi)$ could be at the Planck scale), then we can analyse the potentiais separately. When the universe is more or less at the Planck scale, we can neglect the contribution coming from $V(\phi)$. The dynamical behaviour of the scalar fields are the same as in Case A, i.e inflation is driven by the $R^{2}$ term. However, after inflation, when the universe evolves into the potential "zero" line, the contribution from $V(\phi)$ becomes important.

Hereafter we shall discuss the evolution of the universe after such a stage. A relation similar to (3.6) can be found between $\psi$ and $\phi$, and using it we can rewrite the action and 
the potential $\hat{U}(\phi, \psi)$ as

$$
\begin{aligned}
S & =\int d^{4} x \sqrt{-\hat{g}}\left[\frac{1}{2 \kappa^{2}} R(\hat{g})-\frac{1}{2} \cdot \frac{1-\left(\phi / \tilde{\phi}_{c}\right)^{2}}{\left(1-\left(\phi / \phi_{c}\right)^{2}\right)^{2}}(\dot{\nabla} \phi)^{2}-\hat{i}\right] \\
\hat{U} & =\frac{1}{\left(1-\left(\phi / \phi_{c}\right)^{2}\right)^{2}} V(\phi)
\end{aligned}
$$

where $\tilde{\phi}_{c} \equiv\left[\kappa^{2} \xi(1-6 \xi)\right]^{-1}$ as in [13]. This thecry is the same as one with non-minimal coupling to gravity and has already been investigated in [13]. There it was found that inflation only occurs if $\xi<0$. In particular, if $\xi$ s negative and $V(\phi)$ contains a $\phi^{4}$ term, then the model has two inflationary periods, providing a realization of the double inflation proposed by Turner and Silk [15] (see also [6]). We would like to point out that generally one of the difficulties of Planck scale inflation is that a closed universe may collapse more or less in a Planck time before reaching the GUT stage, however the Planck scale potential is troublesome because it produces too large den;ity perturbations. If, on the other hand, we find double inflation (one at Planck scale, the other at GUT scale), we can solve this problem. The first inflation will prevents the uriverse from collapsing, while the second one would guarantees the present universe isotropy and homogeneity on the large scale, while providing the appropriate density perturbations.

As a special case of this model we will inves igate the induced gravity model with an $R^{2}$ term (no linear term in $R$ ). Here the conformal factor is given by

$$
\omega=\frac{1}{2} \ln \left[\kappa^{2} \epsilon \phi^{2}+2 \alpha R\right]
$$

and the putential in t!ie $\hat{g}$-wor.d is

$$
\hat{U}(\phi, \psi) \equiv \frac{1}{8 \alpha \kappa^{2}}\left[1-\kappa^{2} \epsilon \phi^{2} e^{-\sqrt{\frac{2}{3}} \kappa \psi}\right]^{2}+e^{-\sqrt{\frac{2}{3}} \kappa \psi} V(\phi)
$$

where $V(\phi)=\frac{\lambda}{8}\left(\phi^{2}-\eta^{2}\right)^{2}$. Again for a similitr choice of parameters we show $\hat{L}(\phi, \psi)$ in Fig. 4. We find that double inflation is also present for exactly the same reasons as mentioned earlier. The first inflation occurs due to the curvature squared term. At the end of this period the potential $V(\phi)$ becomes important and since its shape is the same 


\section{ORIGNAL PAGE IS \\ OF. POOR QUALITY}

as that shown in Fig.1, we find a second inflation, this could be exponential or power law depending on exactly where it lands.

\section{Concluding Remarks}

In this paper, we have considered a renormalizable theory, which consists of curvature square and non-minimally coupled terms. We have shown that by using conformal transformations on the metric these theories can be converted into the normal gravitational theory in minimal coupling with a set of scalar fields. These theories are much simpler to analyze by using elementary techniques about the motion of point particles in a given potential than the original theory. The general conclusion is that anisotropic model (and isotropic ones) undergo inflation in these theories without having to fine tune parameters. The general feature is the fact these scalar fields have potentials (in the transformed world) that are extremely flat for large positive values of the fields giving rise to exponential inflation or have the right curvature to produce power law inflation for large negative values of the fields.

The conclusion was reached not by solving the evolution equations for these fields but rather by showing that the energy monerium tensor of these theories satisfies the strong and dominant energy conditions and then invoking the No Hair Conjecture.

Wc would like to finish with a few remarks:

(i) Although the analy'sis in the fictitious $\hat{g}$-world is easier than in the original $g$-world, we always have to return to the original system in order to know what is happening tc our model.

(ii) The use of conformal transformations is inconsequential because the analysis is basically of a classical nature. However, if we were interested in quantum (or semiclas- 
sical) process, like calculating density perturbetion in these models, the two classically equivalert systems might not (and probably are: not) equivalent any longer.

(iii) We have not consider Weyl curvature sq uare terms because in that case our simple transformation breaks down (remember that the Weyl tensor is conformally invariant) and so it is impossible to get rid of this term in the action.

\section{Acknowledgements}

This work was supported in part by the DOE and by the NASA at Fermilab. K.M. acknowledges Rocky Kolb and the Theoretical Astrophysics Group for their hospitality at Fermilab, where most of this work was done. He also thanks the Yamada Science Foundation for financial support to visit Fermilab. 
ORIGINAL PAGE IS

OF POOR QUALITY

\section{Figure Caption}

Fig.1 The potential $\dot{V}(\psi) \equiv V_{0}\left[1-\exp \left(-2 \sqrt{\frac{\epsilon}{1+6 e}} \kappa \psi\right)\right]^{2}$ with $V_{0}=\frac{\lambda}{8 \kappa^{4} e^{2}}$. The special choice $\eta=1, \epsilon=\frac{1}{4}, \kappa=1$ has been made.

Fig. 2a View from above of the potential (3.4) for the case $V(\phi)=0$ and $\xi=0.01$. Both the flat plateau and the minimum are clearly seen.

Fig. 2b View from below. Here the minimum of the potential is clearly shown.

Fig. 3a View from above of the potential (3.4) when $V(\phi)=-\frac{1}{2} m^{2} \phi^{2}+\frac{1}{4} \lambda \phi^{4}$. in this particular case we took $\xi=0.005, m^{2}=10, \lambda=0.1$ in order to highlight some of the features.

Fig. 3b View from below

Fig. 4 The potential 3.11with $\xi=0.1, \eta^{2}=33.33, \lambda=0.6$ in order to highlight some of the features. 


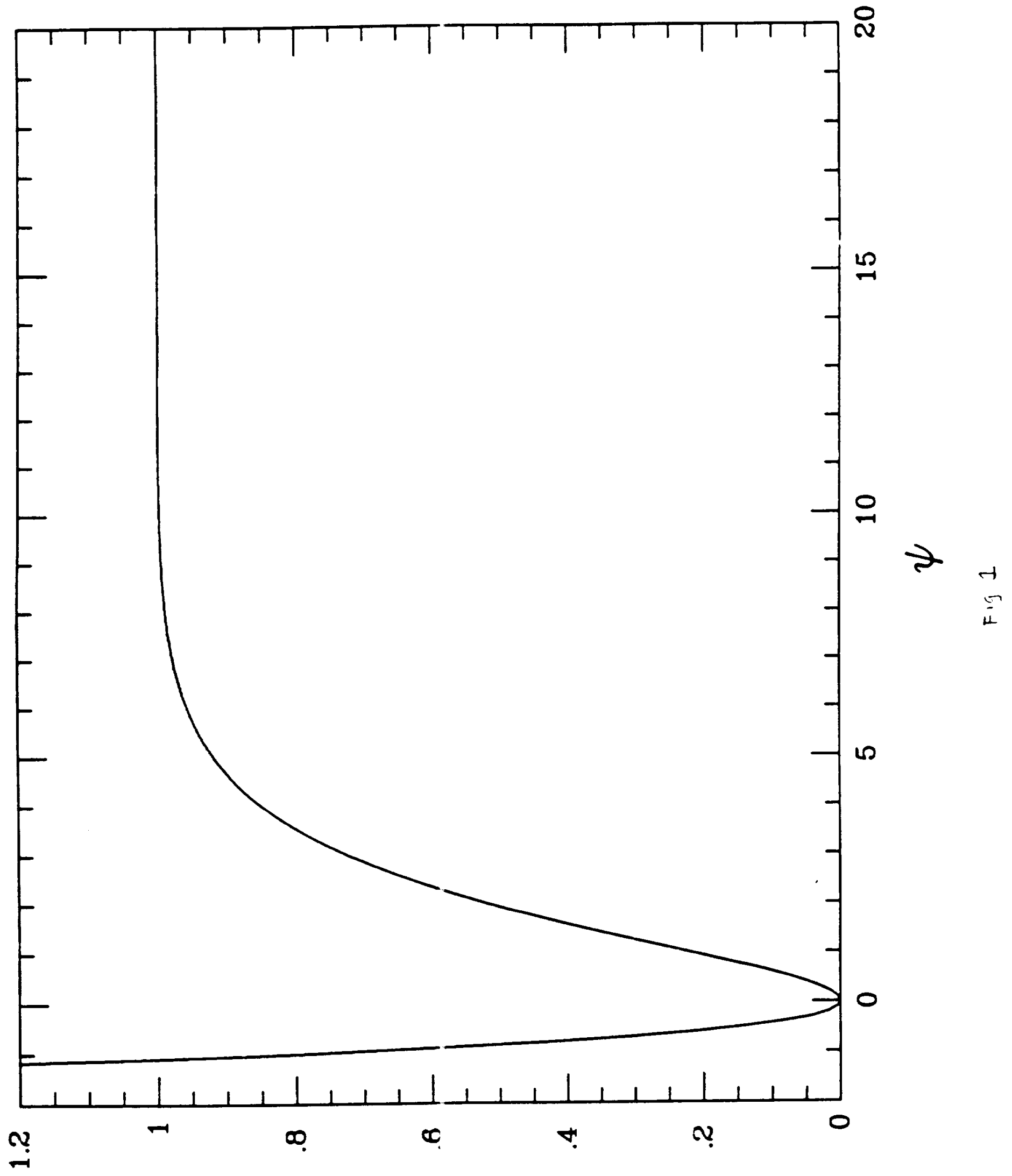




\section{ORIGINAL PAGE IS \\ OF POOR QUALITY}

\section{References}

[1] A.H. Guth, Phys. Rev. D23, 347 (1981)

K. Sato, Mon. Not. R. astr. Soc. 195(1981) 467; Phys. Lett. 99B (1981) 66.

A.Albrecht and P.J. Steinhardt Phys. Rev. Lett. 48,1220 (1982)

A.D. Linde, Phys. Lett. 108B, 389 (1982) ; Phys. Lett. 129B, 177 (1983)

M.S. Turner, The Inflationary Paradigm, Proceedings of the Cargèse School on Fundamental Physics and Cosmology, edited by J. Audouze and J. Tran Thanh Van, Editions Frontieres, Gif-Sur-Yvette (1985).

[2] A.A. Starobinskii, Phys. Lett. 91B (1980) 99; Sov. Astron. Lett. 10 (1984) 135.

[3] M.B. Mijić, M.S. Morris and W.-M. Suen, Phys. Rev. D34, 10(1986),2934

S.W. Hawking and J.C. Luttrell, Nucl. Phys. B247, 280 (1984).

4] A.A. Starobinskii and H.-J. Schmidt, Class. Quantum Grav. 4, 695 (1987)

H.-J. Schmidt, GDR Academy of Sciences (1987).

[5] K. Maeda, Phys. Rev. D37, 858 (1988).

[6] M. Mijić and J.A. Stein-Schabes, Phys. Lett. B203, 353 (1988)

7] J.D. Barrow and A.C. Ottewill, J. Phys. A. 16, 2757 (1983)

J.D. Barrow, Nucl. Phys. B296,697(1988).

[8] R.W. Wald, Phys. Rev. D28, 2118 (1983)

L.G. Jensen and J.A. Stein-Schabes, Phys. Rev. D34,931 (1986)

M.S. Turner and L. Widrow, Phys. Rev. Lett. 57, 2237 (1986) and Nature 326,206 (1987)

G. Steigman and M.S. Turner, Phys. Lett 128B, 295 (1983)

I.Moss and V.Sahni, Phys. Lett. 178, 159 (1986).

[9] L.G. Jensen and J.A. Stein-Schabes, Phys. Rev. D35,11.5 (1937).

J.D. Barrow and J.A. Stein-Schabes, Phys. Lett. 103A, 315 (1984)

J.D. Barrow and O. Grøn, Phys. Lett. 182B, 25 (1986)

J. A. Stein-Schabes, Phys. Rev. D35, 2345 (1987).

[10] V.A. Belinsky, L.P. Grishchuk, I.M. Khalatnikov and Ya. B. Zeldovich, Phys. Lett. B155, 232 (1985).

V.A. Belinsky and I.M. Khalatnikov, Trieste-Preprint IC $/ 86 / 355$.

A.D. Lirde, Phys. Lett. B162, 281 (1985).

G.W. Gibbons, S.W. Hawking and J.M. Stewart, Nucl. Phys. B281, 736(199i).

D.N. Page, Phys. Rev. D36, 1607 (1987).

[11] K. Stelle, Phys. Rev. D 16953 (1977)

L.S. Brown and J.C. Collins, Ann. Phys. 130,215 (1980)

S. Adler, Rev. Mod. Phys. 54, 729 (1982)

L. Smolin, Nucl. Phys. B160 253, (1979).

N.H. Barth and S.M. Christensen, Phys. Rev. D28, 1876 (1983). 


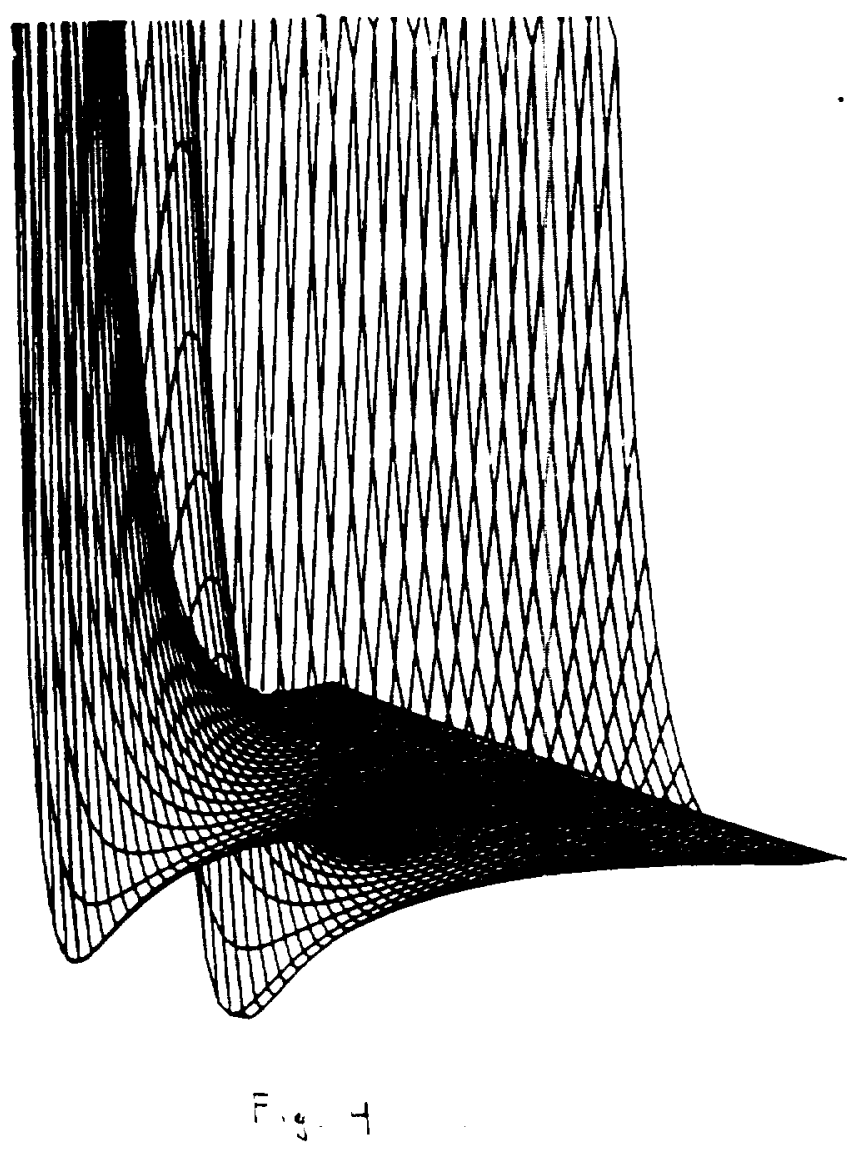

OF POOR QUALIT' 
[12] A. Zee, Phys.Rev.Lett. 42, 417 (1979)

F.S Acceta, D. J. Zoller and M.S. Turner, Phys. Rev. D31,3046 (1985)

[13] T. Futamase and K. Maeda,preprint, UTAP-62/87 (1987).

[14] K. Maeda,preprint, UTAP-75/88(1988)

[15] M.S. Turner and J. Silk, Phys. Rev. D35,419 (1987).

[16] A.A. Starobinskii, Sov. Astron. Lett. 7,36(1981).

[17] B. Whitt, Phys. Lett. 145B, 176 (1984). 
ORIC ANAL

OF pOOR grins:

\begin{tabular}{|c|c|c|c|c|c|c|}
\hline \hline$\Lambda$ & $\kappa_{0}$ & $b_{0} C_{\mu \nu \rho \sigma} C^{\mu \nu \rho \sigma}$ & $c_{0}$ & $g_{\mu,}$ & Inflation & Ref. \\
\hline \hline 0 & 1 & 0 & $\neq 0$ & $B^{2}(t)$ & $y$ & {$[2],[3],[7]$} \\
\hline 0 & 1 & 0 & $\neq 0$ & $\gamma_{i j}(t) \omega^{i}\left(x^{k}\right) \omega^{j}\left(x^{\alpha}\right)$ & $y$ & {$[5],[6]$} \\
\hline$\Lambda$ & 1 & 0 & $\neq 0$ & $\gamma_{i j}(t) \omega^{i}\left(x^{x}\right) \omega^{j}\left(x^{\alpha}\right)$ & $y$ & {$[6]$} \\
\hline 0 & 1 & 0 & $\neq 0$ & $e^{2 H\left(x^{\alpha}\right) t}\left(a_{\mu \nu}\left(x^{\alpha}\right)+\sum b_{\mu \nu}^{(n)} e^{-n H\left(x^{\alpha}\right) t}\right)$ & $y$ & {$[4]$} \\
\hline 0 & 0 & 0 & $\neq 0$ & $e^{2 H\left(x^{\alpha}\right) t}\left(a_{\mu \nu}\left(x^{\alpha}\right)+\sum b_{\mu \nu}^{(n)} e^{-n H\left(x^{\alpha}\right) t}\right)$ & $y$ & {$[4]$} \\
\hline 0 & 0 & $\neq 0$ & $\neq 0$ & $e^{2 H\left(x^{\alpha}\right) t}\left(a_{\mu \nu}\left(x^{\alpha}\right)+\sum b_{\mu \nu}^{(n)} e^{-n H\left(x^{\alpha}\right) t}\right)$ & $y$ & {$[4]$} \\
\hline \hline
\end{tabular}

Table 1: $\mathcal{L}_{\phi}=\xi=0$

\begin{tabular}{|c|c|c|c|c|c|c|}
\hline \hline$\kappa_{0}$ & $b_{0} C_{\mu \nu \rho \sigma} C^{\mu \nu \rho \sigma}$ & $c_{0}$ & $\xi$ & $g_{\mu \nu}$ & Inflation & Ref. \\
\hline \hline 0 & 0 & 0 & $0<\xi<<1$ & $B^{2}(t) \eta_{\mu \nu}$ & $y$ & {$[12]$} \\
\hline 1 & 0 & 0 & $<10^{-3}$ & $B^{2}(t) \eta_{\mu \nu}$ & $y$ & {$[13]$} \\
\hline 0 & 0 & $\neq 0$ & $\neq 0$ & $\gamma_{i j}(t) \omega^{i}\left(x^{\alpha}\right) \omega^{j}\left(x^{\alpha}\right)$ & $y$ & $* * *$ \\
\hline 1 & 0 & $\neq 0$ & $\neq 0$ & $\gamma_{i,},(t) \omega^{i}\left(x^{\alpha}\right) \omega^{j}\left(x^{\alpha}\right)$ & $y$ & $* * *$ \\
\hline
\end{tabular}

Table 2: $\mathcal{L}_{\phi} \neq \|, \Lambda=0$ 


$$
4
$$




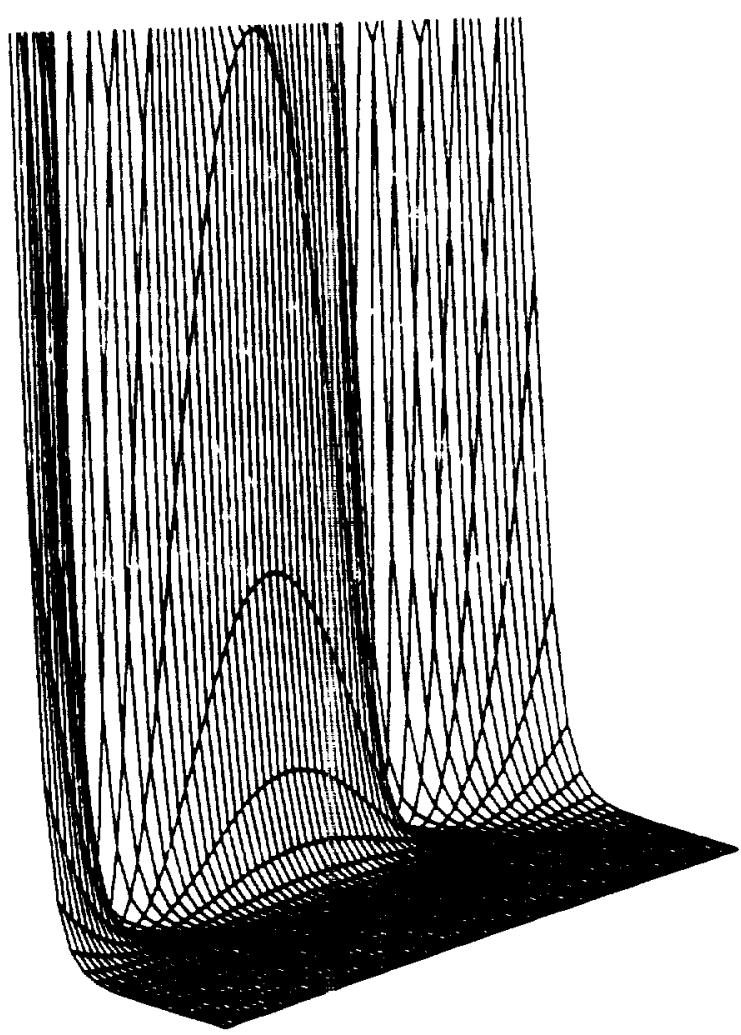

Fig. $3 a$
OR!GR!

OF PCONA

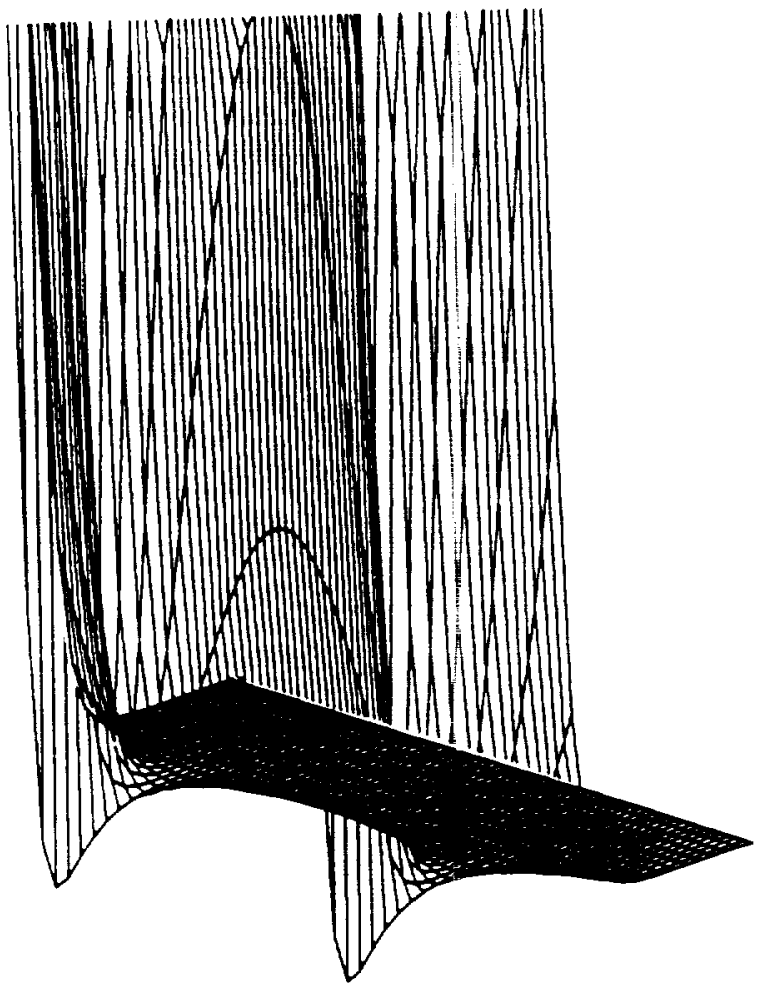

Fig $3 b$ 
